\title{
The encoding of category-specific versus nonspecific information in human inferior temporal cortex
}

\author{
Bingbing Guo \& Ming Meng \\ Dartmouth College, Department of Psychological and Brain Sciences, 6207 Moore Hall, \\ Hanover, NH 03755, USA
}

Corresponding address:

Ming Meng

Dartmouth College

Department of Psychological and Brain Sciences

6207 Moore Hall

Hanover, NH 03755

USA

Email:ming.meng@dartmouth.edu

Phone: 001-603-646-9129

Fax: 001- 603-646-1419 


\begin{abstract}
Several brain areas in the inferior temporal (IT) cortex, such as the fusiform face area (FFA) and parahippocampal place area (PPA), are hypothesized to be selectively responsive to a particular category of visual objects. However, how category-specific and nonspecific information may be encoded at this level of visual processing is still unclear. Using $\mathrm{fMRI}$, we compared averaged BOLD activity as well as multi-voxel activation patterns in the FFA and PPA corresponding to high-contrast and low-contrast face and house images. The averaged BOLD activity in the FFA and PPA was modulated by the image contrast regardless of the stimulus category. Interestingly, unlike the univariate averaged BOLD activity, multi-voxel activation patterns in the FFA and PPA were barely affected by variations in stimulus contrast. In both the FFA and PPA, decoding the categorical information about whether participants saw faces or houses was independent of stimulus contrast. Moreover, the multivariate pattern analysis (MVPA) results were highly stable when either the voxels that were more sensitive to stimulus contrast or the voxels that were less sensitive were used. Taken together, these findings demonstrate that both category-specific (face versus house) and nonspecific (image contrast) information is available to be decoded orthogonally in the same brain areas (FFA and PPA), suggesting that complementary neural mechanisms for processing visual features and categorical information may occur in the same brain areas but respectively be revealed by averaged activity and multi-voxel activation patterns. Whereas stimulus strength, such as contrast, modulates overall activity amplitudes in these brain areas, activity patterns across populations of neurons appear to underlie the representation of object category.
\end{abstract}

Key Words:

Face processing; object recognition; visual contrast; inferior temporal cortex; multivariate pattern analysis; fMRI 


\section{Introduction}

The inferior temporal (IT) cortex is known to play an important role in visual categorization and recognition (Miller et al., 1991; Hung et al., 2005). The early visual areas in the occipital lobe are thought to process low-level features (Hubel and Wiesel, $1962,1965)$, while the synthesis of these features to form high-level object representations is presumed to occur in the IT cortex (Felleman and Van Essen, 1991; DiCarlo and Maunsell, 2000; Yamins et al., 2014). Consistent with this notion, previous neurophysiological and neuroimaging studies have shown object-selective properties of IT neurons in nonhuman primates (Gross et al., 1972; Desimone et al., 1984; Tsao et al., 2003; Pinsk et al., 2005; Tsao et al., 2006; Freiwald et al., 2009; Freiwald and Tsao, 2010). Through fMRI measurements of BOLD activity, a few regions in the human IT cortex have also been identified to be selectively responsive to some particular categories of objects (Kanwisher, 2010). Most notably, an area in the lateral fusiform gyrus has been hypothesized to be selectively responsive to images of faces (the fusiform face area, FFA) (Kanwisher et al., 1997), whereas the parahippocampal place area (PPA) (Epstein and Kanwisher, 1998) is hypothesized to respond to images of places more strongly than to images of faces.

How would perceptual processing lead to categorical representations in the IT cortex? Note that visual features such as contrast are not specific to any object categories and can vary continuously in a physically measurable dimension. There may be a detection threshold, but if, for example, neurons in the FFA were truly the "face cells", one would assume that the response function of these neurons should be invariant to low-level visual features such as contrast, as long as the contrast is above the detection threshold and the face stimuli can be recognized. If, however, the nonspecific information modulates responses in the FFA, some other representation mechanism should be able to encode the category-specific information that is invariant to low-level features at a later stage. Indeed, a recent fMRI study found that lower-level visual features significantly modulate the averaged BOLD activation in the FFA (Yue et al., 2011). This result suggests that averaged BOLD activity in the FFA potentially reflects stimulus properties that are non-specific to faces, and may not be directly used to encode category-specific information that should be invariant to low-level features. It remains unknown whether the contrast of non-face images may also modulate FFA activity, or whether other brain areas in the IT cortex (e.g. PPA) may be modulated by stimulus contrast.

Alternative to the hypothesis that object categories are encoded by categoryselective neurons is the possibility that the encoding of object categories is accomplished through the activity of a distributed population of neurons (Gochin et al., 1994; Haxby et al., 2001). If a distributed ensemble of neurons in the IT cortex encodes faces (Haxby et al., 2000; Nestor et al., 2011), activation patterns would reflect the categorical representation of faces better, instead of univariate averaged BOLD activity. 
On one hand, signal strength of the input (e.g. high contrast) may drive neurons to fire more vigorously, leading to overall increased BOLD activity (Heeger et al., 2000). On the other hand, activation patterns do not have to change, providing the possibility for the encoding of object categories. Hypothetically, the transition of local averaged neuronal activity to an activation pattern of distributed populations of neurons would fit naturally with the non-linear transformation of encoding from continuous visual features to discrete categorical information. We test this hypothesis in the present study by comparing univariate averaged BOLD activity with the results from multivariate pattern analysis (MVPA) of fMRI activity. MVPA uses machine-learning techniques (e.g., PyMVPA, see Hanke et al., 2009) to discriminate multi-voxel patterns of brain activity corresponding to different experimental conditions (e.g. seeing faces versus seeing houses). Whereas univariate averaged BOLD activity may correlate with the local averaged neuronal activity (Bandettini and Ungerleider, 2001; Heeger and Ress, 2002; Logothetis et al., 2001), activation patterns of a distributed population of neurons can be revealed by the MVPA (e.g., Haxby et al., 2001). If categorical representation occurs through encoding by populations of neurons in the IT cortex, we expect that the decoding through MVPA should be independent of stimulus contrast, despite that averaged BOLD activity being significantly modulated by stimulus contrast (e.g. see Yue et al., 2011). If, however, the encoding in the IT cortex is not independent of stimulus contrast, MVPA results would also be modulated by stimulus contrast.

\section{Materials and Methods}

Seventeen healthy adults, (mean age $=26 \mathrm{y}$, seven females) with normal or correctedto-normal visual acuity, participated in this study. The data from five participants were excluded from further analysis because of excessive head motion (> $3 \mathrm{~mm}$ ). All participants gave written informed consent. The study was approved by the Committee for the Protection of Human Subjects at Dartmouth College.

\section{MRI Acquisitions}

Scanning was performed on a 3.0 T Philips Achieva Intera scanner with a 32-channel head coil at the Dartmouth Brain Imaging Center. The BOLD signals were collected using an echo-planar imaging (EPI) sequence $(\mathrm{TR}=2000 \mathrm{~ms}, \mathrm{TE}=35 \mathrm{~ms}$, flip angle $=$ $90^{\circ}, \mathrm{FOV}=240 \mathrm{~mm}$, voxel size $=3 \times 3 \times 3 \mathrm{~mm}, 35$ slices). For each participant, a highresolution magnetization-prepared rapid-acquisition gradient echo (MPRAGE) anatomical scan was acquired at the end of the scan session (TR $=8.2 \mathrm{~ms}$, TE $=3.8$ $\mathrm{ms}$, flip angle $=8^{\circ}, \mathrm{FOV}=240 \mathrm{~mm}$, voxel size $=1 \times 1 \times 1 \mathrm{~mm}, 222$ slices). During the EPI scans, visual stimuli were presented to a screen located at the back of the scanner via a LCD projector (Panasonic PT-D4000U, $1024 \times 768$ pixels resolution) using MATLAB 2011b with Psychtoolbox (Brainard 1997; Pelli 1997). Participants viewed the 
stimuli using a mirror placed within the head coil. Stimuli subtended a visual angle of $8.7^{\circ}$.

\section{ROI Localizer runs}

To localize the FFA and PPA as the regions of interest (ROI), visual stimuli were chosen from an independent set of gray-scale images of faces and houses. The localizer scans consisted of an alternating block design, with 5 stimulation blocks presenting face images and 5 stimulation blocks presenting house images interleaved with 16-s periods of a blank screen with a fixation cross in the center. Each stimulation block was also 16s long. In total, each localizer scan was 336-s long, consisting of 11 periods of fixation and 5 blocks for each of the two stimulation categories. In each stimulation block, 16 faces (or houses) were presented (500 ms per image, with a 500-ms interstimulus interval). Each participant completed two localizer scans. During these scans, participants performed a one-back task in which they were asked to make a key-press whenever an image was repeated consecutively.

\section{Experimental runs}

The stimuli set included gray-scale images of eight conditions: low-contrast faces, highcontrast faces, low-contrast houses, high-contrast houses and four conditions for a separate study that is not directly relevant. The high contrast level was defined with root mean square $(R M S)=0.25$ in normalized unit, whereas the low contrast level RMS = 0.025 in normalized unit. Corresponding contrasts of faces and houses were made equal by using the SHINE toolbox (Willenbockel et al., 2010). The experimental scans consisted of an alternating block design, with 16-s blocks of stimulation interleaved with 16-s of fixation periods. In total, each experimental scan was 272-s long, consisting of 9 fixation periods and 1 stimulation block for each of the eight conditions. In each stimulation block, 8 images from a condition were presented (1700 ms per image, with 300-ms interstimulus interval). Each participant completed 9-10 experimental scans. During these scans, participants performed a color detection task in which they were asked to press a button whenever the entire image was presented in red for $200 \mathrm{~ms}$ at random times across every stimulation block. The purpose of this task was to ensure that participants had been attentive to the stimuli.

\section{Data analysis}

AFNI (http://afni.nimh.nih.gov/afni) was used for preprocessing the MRI data. EPIs were motion corrected to the image acquired closest to the anatomical images, spatially smoothed with a 4-mm full width at half maximum filter (FWHM), and temporally filtered to remove baseline drifts. The anatomical images were aligned to the functional images to avoid the additional resampling and interpolation of functional images. 
Data from the localizer scans were further submitted to a General Linear Model (GLM) analysis, which allowed the calculation of beta coefficient values associated with block conditions. ROls were individually defined for each participant based on activation maps from the GLM analysis. Each ROI was defined as a continuous cluster of activated voxels corresponding to the following statistical contrasts: the FFA was defined in the right middle fusiform gyrus as responding more strongly to faces than to houses ( $p<10^{-4}$, uncorrected), and the PPA was defined in the right parahippocampal gyrus as responding more strongly to houses than to faces $\left(p<10^{-4}\right.$, uncorrected). To control for any potential confounding effects of ROI size, the FFA and PPA were localized with roughly the same number of voxels ( 40 voxels). We focus on results of $\mathrm{ROls}$ in the right hemisphere here. Results in the left hemisphere are similar, except that by using the same criteria, we were only able to successfully identify the left FFA with $\sim 40$ voxels in eight participants. This is consistent with previous reports about lateralization of the FFAs in humans (e.g., Kanwisher et al., 1997; Behrmann \& Plaut, 2013).

For comparison, we also used an $\mathrm{ROI}$ in the early visual cortex. The Brodmann area 17 (BA17) was localized using an anatomical mask as well as BOLD contrasts during ROI localizer scans. The anatomical mask of BA17 (TT_N27 template) was individually aligned to the anatomical images of each participant. Using the BOLD contrasts acquired through analyzing the ROI localizer scans, activated voxels were localized in the calcarine sulcus that responded more strongly during stimulation blocks than during fixation periods $\left(p<10^{-4}\right.$, uncorrected). The overlapping region of anatomical and functional localization was then defined as the ROI of BA17 in our study. The size of the BA17 ROI was on average 100 voxels.

In each $\mathrm{ROI}$, we further analyzed the brain responses to the four experimental conditions (low-contrast faces, high-contrast faces, low-contrast houses and highcontrast houses) using two different methods. First, for each participant, we extracted the beta coefficient values averaged across all voxels in each ROI to perform a univariate analysis of BOLD contrasts. Further statistical analyses (ANOVAs and Student's T-tests) were conducted to compare the beta values of all the stimulus conditions in each ROI across participants. Secondly, a multivariate pattern analysis was performed using PyMVPA (Hanke et al., 2009). We extracted the beta values associated with experimental conditions of all the voxels in each $\mathrm{ROI}$, removing the mean intensity for each multi-voxel activity pattern. For each participant, the brain response pattern analyses of classification training and testing with linear support vector machines (SVM) were conducted using a leave-one-run-out cross-validation procedure. Furthermore, to evaluate whether stimulus contrast modulates brain response patterns, cross-validations that use low-contrast condition data for training and high-contrast condition data for testing, and vice versa, were also conducted. ANOVAs were then conducted to compare classification accuracies. 


\section{Results}

Fig. 1 A shows examples of the stimuli. Fig. 1B shows ROls in a representative participant. BOLD responses were sorted and averaged corresponding to the four experimental conditions. The results in these ROls from the representative participant are plotted as a function of time in Fig. 1C.

Figure 1 about here

Figure 1. (A) Examples of stimuli. (B) Localization of ROls in a representative participant. The FFA (red), PPA (blue) and BA17 (green) were shown in coronal, sagittal and horizontal views. (C) The averaged BOLD responses in these ROls as a function of time. Error bars represent 1 S.E.M.

\section{Univariate analysis of averaged BOLD response}

Averaged beta values in the FFA, PPA and BA17 corresponding to the four experimental conditions are shown in Fig. 2. Two-way repeated measures ANOVAs were conducted to analyze the effect of object category (face vs. house) and the effect of stimulus contrast. In the FFA, the main effect of object category was highly significant $(F(1,11)=126.040, p<0.001)$, and interestingly, the main effect of stimulus contrast also reached significance $(F(1,11)=6.271, p<0.05)$. The interaction between object category and stimulus contrast was not significant $(F(1,11)=0.061, p=0.809)$. Similar to the FFA, both the main effect of object category $(F(1,11)=107.009, p<0.001)$ and the main effect of stimulus contrast $(F(1,11)=20.939, p<0.01)$ were significant in the PPA, while the interaction between object category and stimulus contrast was not significant $(F(1,11)=2.407, p=0.149)$. For comparison, in the early visual area of $\mathrm{BA} 17$, the main effect of stimulus contrast was highly significant $(F(1,11)=26.447, p<0.001)$, whereas the main effect of object category was not $(F(1,11)=2.373, p=0.152)$. The interaction between object category and stimulus contrast was not significant either $(F(1,11)=0.106$, $p=0.751$ ).

Figure 2 about here

Figure 2. Results of the univariate BOLD responses in the FFA, PPA and BA17. The averaged beta values corresponding to the four experimental conditions (low-contrast faces, high-contrast faces, lowcontrast houses and high-contrast houses) are shown. Error bars represent 1 S.E.M. 


\section{Multivariate pattern analysis}

Results from our univariate analysis were consistent with previous findings, suggesting that faces led to greater responses than houses in the FFA (Kanwisher et al., 1997) while houses led to greater responses than faces in the PPA (Epstein and Kanwisher, 1998). More interestingly, high-contrast faces led to greater brain activation in the FFA than low-contrast faces (cf. Yue et al., 2011). Quite surprisingly, despite faces and houses being clearly differentiable even in our low-contrast conditions, high-contrast stimuli led to greater brain activation in the FFA and PPA than low-contrast stimuli, independent of whether it was a face or a house. Are these results due to the same populations of neurons in the FFA and PPA responding to high-contrast stimuli and lowcontrast stimuli with different firing rates, or due to fewer (and perhaps different) populations of neurons responding to low-contrast stimuli than to high-contrast stimuli? This question cannot be answered by analyzing univariate averaged BOLD responses in these ROls. Therefore, we employed MVPA to examine spatially distributed activation patterns across multiple voxels in the ROls.

Classification confusion matrix. First, brain response pattern analyses of classification training and testing with linear support vector machines (SVM) were conducted using PyMVPA (Hanke et al., 2009) with a leave-one-run-out cross-validation procedure. A confusion matrix was constructed to indicate how often the classifier correctly categorized the multi-voxel activation patterns corresponding to the experimental conditions, and how often it confused the pattern categorization. Fig. 3A shows the confusion matrices in the FFA, PPA, and BA17. Elements of the confusion matrix (color-mapped visualization) show the number of trials that were classified correctly (diagonal) or incorrectly (off-diagonal). The results in the FFA and PPA suggest that multi-voxel activation patterns corresponding to faces were quite distinct from houses, although high stimulus contrast conditions were often confused with low stimulus contrast conditions. For comparison, these effects were not seen in BA17, suggesting that our results in the FFA and PPA are unlikely to be artifactual, driven by some inadvertent regularity in the stimulus presentation blocks.

Classification accuracy. To further quantify whether the activation patterns in the FFA and PPA were comparable for high-contrast vs. low-contrast stimuli, we examined MVPA classification accuracies in the following four situations: 1) the linear SVMs trained with the data corresponding to high stimulus contrast conditions and also tested with the data corresponding to high stimulus contrast conditions using a leave-one-out procedure; 2 ) the linear SVMs trained with the data corresponding to high stimulus contrast conditions but tested with the data corresponding to low stimulus contrast conditions; 3) the linear SVMs trained with the data corresponding to low stimulus contrast conditions but tested with the data corresponding to high stimulus contrast 
conditions; 4) the linear SVMs trained with the data corresponding to low stimulus contrast conditions and also tested with the data corresponding to low stimulus contrast conditions using a leave-one-out procedure. The results are shown in Fig. 3B. To validate whether the classification performance was significantly above chance, we further conducted Monte Carlo permutation-based statistical tests. This method entailed running a classification analysis 1,000 times with randomly permuted experimental condition labels, allowing us to construct null distributions that were used to examine whether a classification accuracy was significantly above chance at an $\alpha$ of $p<0.05$.

In the FFA, the classification accuracy (AC) of high-contrast face vs. house was significantly above chance (mean $\mathrm{AC}=83.41 \%, p<0.05$ ). For the low-contrast face vs. house, the classification accuracy was also significantly above chance (mean $A C=88 \%$, $p<0.01$ ). Similar results were found in the PPA, the classification accuracies for object categories were significantly above chance, with both high stimulus contrast (mean $\mathrm{AC}=83.19 \%, p<0.01$ ) and low stimulus contrast (mean $\mathrm{AC}=81.39 \%, p<0.05)$. Not surprisingly, in the BA17, classification accuracies for object categories were much lower than the results in the FFA and PPA, for both the high-contrast stimuli (mean $\mathrm{AC}=62.89 \%, p=0.059$ ) and low-contrast stimuli (mean $\mathrm{AC}=61.08 \%, p=0.064$ ). More interestingly, classification accuracies of the cross-validations that use the data corresponding to low-contrast stimuli for training and the data corresponding to highcontrast stimuli for testing, and vice versa, were also highly robust in the FFA and PPA (FFA: $A C=85.12 \%$ and $87.11 \%$ respectively, $\mathrm{PPA}: \mathrm{AC}=84.64 \%$ and $75.72 \%$ respectively, all significantly above chance, $p s<0.05)$, suggesting the activation patterns in these brain areas were mainly driven by object information regarding stimulus category (i.e. face vs. house) and independent of stimulus contrast levels. For comparison, in the BA17, the classification accuracies with the cross-validations that used the data corresponding to low-contrast stimuli for training and the data corresponding to high-contrast stimuli for testing (mean $A C=54.97 \%, p=0.18$ ), and vice versa (mean $A C=51.91 \%, p=0.21$ ), were not significantly above chance.

Finally, we examined whether MVPA could directly differentiate the activation patterns driven by high-contrast stimuli vs. low-contrast stimuli. Fig. $3 \mathrm{C}$ shows the classification accuracies respectively for high-contrast vs. low-contrast regardless of the stimulus category, high-contrast face vs. low-contrast face, and high-contrast house vs. low-contrast house. In the FFA, the results were not significantly different from chance (mean $\mathrm{AC}=54.54 \%$ for high-contrast vs. low-contrast regardless of the stimulus category, $p=0.26$; mean $A C=51.68 \%$ for high-contrast face vs. low-contrast face, $p=0.12$; mean $A C=57.77 \%$ for high-contrast house vs. low-contrast house, $p=0.20$ ). The results were not significantly different from chance in the PPA either (mean $A C=57 \%$ for high-contrast vs. low-contrast regardless of the stimulus category, $p=0.27$; mean $\mathrm{AC}=60.83 \%$ for high-contrast face vs. low-contrast face, $p=0.19$; mean $\mathrm{AC}=63.76 \%$ for high-contrast house vs. low-contrast house, $p=0.11$ ). However, for comparison, in the 
BA17, the results were marginally significant (mean $A C=64.1 \%$ for high-contrast vs. lowcontrast regardless of the stimulus category, $p=0.052$; mean $A C=67.69 \%$ for highcontrast face vs. low-contrast face, $p=0.07$; mean $A C=63.14 \%$ for high-contrast house vs. low-contrast house, $p=0.07$ ). These results suggest that, unlike univariate averaged BOLD responses, brain activation patterns in the FFA and PPA were barely affected by stimulus contrast.

Figure 3 about here

Figure 3. Results of MVPA in the FFA, PPA and BA17. (A) Confusion matrices to the four experimental conditions. (B) Pattern classification accuracies of the experimental conditions corresponding to object categories. (C) Pattern classification accuracies of decoding stimulus contrast. Error bars represent 1 S.E.M. * indicates $p<0.05,{ }^{* *}$ indicates $p<0.01$.

It is worth noting that our findings, described above, were not altered with using larger ROls. The larger ROls were localized by growing the size of the 40 -voxel FFA/PPA to $\sim 100$ voxels through relaxing statistical threshold for each participant (maximum $p<0.05$, uncorrected). Moreover, we evenly divided 100 voxels in the larger ROls into two sets based on sensitivity to stimulus contrast. To further evaluate whether the decoding of categorical information through MVPA is independent of contrast sensitivity or not, we compared MVPA results in each of the larger ROI using the set of top 50 voxels that were most sensitive to stimulus contrast (HCFFA and HCPPA), with the set of bottom 50 voxels that were least sensitive to stimulus contrast (LCFFA and LCPPA). As shown in Fig. 4A and Fig. 4D, spatial distributions of these two sets of voxels appear to be quite random. Future studies are needed to further investigate the distributions. Nonetheless, contrast sensitivities of these two sets were highly significantly different (Fig. 4A, HCFFA vs. LCFFA, $t(11)=7.307, p<0.001$; Fig. 4D, HCPPA vs. LCPPA, $t(11)=7.163, p<0.001)$. These results are consistent with the hypothesis that the activation pattern may not cohesively encode stimulus contrast. Moreover, both confusion matrices and classification accuracies are highly comparable by using the set of voxels that were more sensitive to stimulus contrast versus the set of voxels that were less sensitive. A repeated measure ANOVA reveals that for MVPA classification accuracies in the FFA, the main effect of using high sensitive to contrast (HCFFA) voxels versus low sensitive to contrast (LCFFA) voxels was not significant $(F(1,11)=0.894, p=0.365)$. In the PPA, the main effect of using high sensitivity to contrast (HCPPA) voxels versus low sensitivity to contrast (LCPPA) voxels was significant $(F(1,11)=19.308, p<0.01)$, however, the classification accuracies using 
HCPPA were lower than LCPPA. This is consistent with the hypothesis that MVPA results for decoding object categories would not be improved by using high contrast stimuli. No other main effects or interactions were significant $(F s<2.585, p s>0.136)$.

Figure 4 about here

Figure 4. Results of MVPA in the FFA using high sensitive to contrast voxels (HCFFA), low sensitive to contrast voxels (LCFFA), and in the PPA using high sensitive to contrast voxels (HCPPA), low sensitive to contrast voxels (LCPPA). (A) Spatial distributions of the HCFFA (red) and the LCFFA (green) on the cortical surface of one representative participant (left panel). The averaged effect of contrast in the HCFFA and LCFFA (right panel). (B) Confusion matrices to the four experimental conditions in the HCFFA and LCFFA. (C) Pattern classification accuracies of the experimental conditions corresponding to object categories in the HCFFA and LCFFA. (D) Spatial distributions of the HCPPA (red) and the LCPPA (green) on the cortical surface of one representative participant (left panel). The averaged effect of contrast in the HCPPA and LCPPA (right panel). (E) Confusion matrices to the four experimental conditions in the HCPPA and LCPPA. (F) Pattern classification accuracies of the experimental conditions corresponding to object categories in the HCPPA and LCPPA. Error bars represent 1 S.E.M. * indicates $p<0.05,{ }^{* *}$ indicates $p<0.01,{ }^{* * *}$ indicates $p<0.001$.

\section{Discussion}

Our results suggest complementary neural mechanisms for visual feature analysis and conceptual representation of object category that are respectively revealed by averaged BOLD activity and multi-voxel activation patterns. In the FFA and PPA, categorical information of whether participants see faces or houses can be decoded by using MVPA independent of stimulus contrast. By comparison, the averaged BOLD activity in the FFA and PPA was modulated by the image contrast of both faces and houses. Although the contrast of faces is known to modulate FFA activity (Yue et al., 2011), our study is the first to examine the effect of contrast of both faces and non-face objects, and to compare these effects in the FFA and PPA. More importantly, increasingly convergent evidence suggests that encodings of object categories rely on activation patterns instead of overall averaged activity of brain areas in the IT cortex (Haxby et al., 2001; Cox and Savoy, 2003; for reviews see, Haynes and Rees, 2006; Naselaris et al., 2011; Tong and Pratte, 2012). Our study is the first to directly evaluate whether the activation patterns of these brain areas are independent of stimulus contrast. Our findings suggest that both categorical-specific (face versus house) information and nonspecific (image contrast) information are represented in the FFA and PPA. Moreover, the encoding of object categories may rely on activation patterns across distributed populations of neurons, which are dissociable from the averaged BOLD activity that presumably corresponds to local averaged neuronal activity. Meanwhile, despite that stronger stimulus contrast would lead to greater averaged BOLD activity, the contrast does not appear to change activation patterns in the FFA and PPA. 
Why would stimulus contrast modulate the averaged BOLD activity in the FFA and PPA? One possibility is that low-level features such as stimulus contrast can be considered as a reflection of the signal strength of inputs to the visual system. Just as increased contrast leads to increased firing rates of neurons in the early visual areas (Albrecht and Hamilton, 1982; Sclar et al., 1990), the increased activity of these neurons that project to the FFA and PPA would lead to increased univariate averaged BOLD activity corresponding to local averaged neuronal activity in the FFA and PPA. Nonetheless, our results suggest that the averaged BOLD activity in the FFA and PPA cannot encode the category-specific information independently of stimulus contrast. Some non-linear transformation mechanisms would be necessary to construct the representation of discrete object categories by synthesizing the univariate averaged BOLD activity corresponding to continuous visual features such as contrast.

Our MVPA results indicate that decoding of the discrete category-specific information (i.e. face vs. house) from activation patterns in the FFA and PPA is independent of the non-specific stimulus contrast. One possibility is that there are heterogeneous patches in the FFA and PPA, e.g., studies with non-human primates reported that certain face patches were invariant to low-level features whereas other patches were not (Freiwald and Tsao, 2010). However, this hypothesis could not explain our reliable MVPA results with using either contrast sensitive or insensitive voxels (Fig. 4). Instead, given the notion that an ensemble of neurons, instead of "grandmother cells", may encode the concept of an object, activity patterns across populations of neurons may underlie the invariant representation of object categories (Gochin et al., 1994; Haxby et al., 2001; Pasupathy and Connor, 2002; Averbeck et al., 2006). Whereas decoding of the object categories relies on activation patterns in the FFA and PPA, our results also confirm previous reports suggesting critical roles of the FFA and PPA in processing faces and houses (Kanwisher et al., 1997; Epstein and Kanwisher, 1998). More interestingly, however, the multivariate activation pattern does not rely on the linear summation of local averaged neuronal activity, allowing the nonlinear transformation of binding continuous visual features to discrete objects (James, 1890; Treisman and Gelade, 1980). Consistent with our results, object categorization based on activation patterns in IT may therefore be dissociated with activity in early areas that correlates with the processing of visual features such as contrast, color, or image-level properties that can be modeled by Gabor-jets (Lades et al., 1993; Biederman and Kalocsai, 1997; cf. Olman et al., 2004). Although the binding problem itself is still far from being solvable, future studies that follow our approach to investigating the relationship between local averaged neuronal activity and brain activation patterns would likely provide further insights to understand the neural mechanism underlying ensemble encoding.

Given that analyzing univariate activation versus multi-voxel pattern of our data led to qualitatively different results, what could be inferred from the differences about 
possible relationships between univariate analysis and MVPA, both of which are cornerstones to interpreting $\mathrm{fMRI}$ data? Because of the intrinsically advanced computational power of MVPA, it is often intuitively thought that MVPA can provide more information about brain functions than univariate analysis. However, our results provide a marked example that demonstrates the power of univariate analysis for averaged BOLD activation when a brain region is driven by non-specific visual properties of the stimuli (e.g. contrast). This is analogical to previous findings that demonstrated the effect of contrast in human MT (Tootell et al., 1995), whereas the more specific information of motion directions was decoded with MVPA (Kamitani and Tong, 2006). In general, it has been proposed that univariate activation corresponds to a particular processing mental function while multi-voxel patterns correspond to mental representations (Mur et al., 2009; Coutanche, 2013). Our results support this notion, complementing to the most recent discussions about possible relationships between univariate analysis and MVPA (Davis et al., 2014; Tong et al., 2012).

Despite being highly sensitive to the different activation patterns corresponding to faces and houses, MVPA revealed essentially no effect of contrast on activation patterns in the FFA and PPA. Although the difference of contrast levels in our study led to significant effects of the averaged BOLD in the FFA and PPA, it remains possible that a larger difference in contrast than that of our current study could also lead to changes in activation patterns. Due to the resolution limits of fMRI, we may never be able to fully rule out the possibility that finer activity patterns could still potentially encode stimulus contrast. In addition to the increased averaged BOLD that may be reflective of local averaged neuronal activity, increased stimulus strength with increased contrast may also lead to, for example, decreased overall variance across populations of neurons. Accordingly, it is also possible that the linear SVMs in our MVPA were not the most sensitive machine-learning tool for detecting differences in the overall variance. Attention has been recently reported to modulate variance of activity across a population of neurons in addition to, and perhaps functionally more important than, averaged neuronal activity (Cohen and Maunsell, 2009). Meanwhile, attentional effects have been previously measured with averaged BOLD to evaluate contrast gain control (Liu et al., 2005). It would be interesting for future studies to compare how featurebased attention in addition to stimulus features (e.g., contrast) may have similar or different effects on averaged BOLD vs. activation patterns corresponding to different object categories.

Finally, our results suggest that cautions should be taken for contrasting univariate averaged BOLD responses to localize face-selective/place-selective areas. Given image-level properties, such as contrast modulate averaged BOLD responses in the IT cortex, precise locations of ROls defined by the univariate contrast between two conditions, such as faces vs. houses, would also be contingent upon the image-level properties. For example, Fig. 5 shows how the results from localizing the FFA and PPA 
in a representative participant may be different if the averaged BOLD responses corresponding to faces and houses with different contrast levels were used. On average, $74 \%$ of the voxels localized by comparing high-contrast faces vs. high-contrast houses overlap with $70 \%$ of the voxels localized by comparing low-contrast faces vs. low-contrast houses in the FFA; $57 \%$ of the voxels localized by comparing high-contrast houses vs. high-contrast faces overlap with $66 \%$ of the voxels localized by comparing low-contrast houses vs. low-contrast faces in the PPA. Since the categorical information, decoded through MVPA, of whether participants see faces or houses would be independent of stimulus contrast, any $\mathrm{ROI}$ that is localized by contrasting univariate averaged BOLD responses may not necessarily be the most appropriate region for any subsequent analysis and interpretation of activation patterns in that brain region.

Figure 5 about here

Figure 5. The spatial distribution on the cortical surface of the FFA and PPA localized by contrasting univariate averaged BOLD responses to faces and houses in a representative participant. Using the same statistical criterion, cortical areas that were localized only through comparing BOLD responses corresponding to high-contrast faces vs. high-contrast houses were shown in red; areas that were localized only through comparing BOLD responses corresponding to low-contrast faces vs. low-contrast houses were shown in green; overlapping areas that can be localized by either using high-contrast faces vs. high-contrast houses or using low-contrast faces vs. low-contrast houses are shown in yellow.

In sum, our results provide novel neuroimaging evidence for an ensemble coding model, instead of a hierarchical model for representations of object categories. A hierarchical model would assume that visual processing starts from analyzing simple features and then combines these features to form complex objects. Different brain areas along the visual pathways could presumably accomplish these stages of processing respectively. However, our results demonstrate that both category-specific information and non-specific visual features are represented in the FFA and PPA. Moreover, our results suggest category-specific information and non-specific visual features are encoded in qualitatively disassociated ways: whereas low-level visual features modulate the averaged BOLD activity, brain activation patterns correlate with encodings of the category-specific information independent of low-level visual features. Despite that the local averaged neuronal activity in the IT may be modulated by lowlevel visual properties, category-specific information is represented in the IT as well, likely through an ensemble across distributed populations of neurons. 


\section{ACKNOWLEDGMENTS}

Work supported by National Science Foundation under Grant No. 1157121. We thank Christine Lu and Nicolina S Mascia for improving the English style. 


\section{References}

Albrecht DG, Hamilton DB (1982) Striate Cortex of Monkey and Cat - Contrast Response Function. Journal of neurophysiology 48:217-237.

Averbeck BB, Latham PE, Pouget A (2006) Neural correlations, population coding and computation. Nat Rev Neurosci 7:358-366.

Bandettini PA, Ungerleider LG (2001) From neuron to BOLD: new connections. Nat Neurosci 4:864-866.

Behrmann M, Plaut DC (2013) Distributed circuits, not circumscribed centers, mediate visual recognition. Trends in cognitive sciences 17: 210-219.

Biederman I, Kalocsai P (1997) Neurocomputational bases of object and face recognition. Philosophical transactions of the Royal Society of London Series B, Biological sciences 352:1203-1219.

Brainard DH (1997). The psychophysics toolbox. Spatial vision 10:433-436.

Cox DD, Savoy RL (2003) Functional magnetic resonance imaging (fMRI) "brain reading": detecting and classifying distributed patterns of fMRI activity in human visual cortex. Neuroimage 19:261-270.

Cohen MR, Maunsell JH (2009) Attention improves performance primarily by reducing interneuronal correlations. Nat Neurosci 12:1594-1600

Coutanche MN (2013) Distinguishing multi-voxel patterns and mean activation: why, how, and what does it tell us? Cognitive, affective \& behavioral neuroscience 13:667-673.

Davis T, LaRocque KF, Mumford JA, Norman KA, Wagner AD, Poldrack RA (2014) What do differences between multi-voxel and univariate analysis mean? How subject-, voxel-, and trial-level variance impact fMRI analysis. Neurolmage, 97:271-283.

Desimone R, Albright TD, Gross CG, Bruce C (1984) Stimulus-selective properties of inferior temporal neurons in the macaque. The Journal of neuroscience : the official journal of the Society for Neuroscience 4:2051-2062.

DiCarlo JJ, Maunsell JHR (2000) Form representation in monkey inferotemporal cortex is virtually unaltered by free viewing. Nat Neurosci 3:814-821.

Epstein R, Kanwisher N (1998) A cortical representation of the local visual environment. Nature 392:598-601.

Felleman DJ, Van Essen DC (1991) Distributed hierarchical processing in the primate cerebral cortex. Cerebral cortex 1:1-47.

Freiwald WA, Tsao DY, Livingstone MS (2009) A face feature space in the macaque temporal lobe. Nat Neurosci 12:1187-1196.

Freiwald WA, Tsao DY (2010) Functional Compartmentalization and Viewpoint Generalization Within the Macaque Face-Processing System. Science 330:845-851.

Gochin PM, Colombo M, Dorfman GA, Gerstein GL, Gross CG (1994) Neural Ensemble Coding in Inferior Temporal Cortex. Journal of neurophysiology 71:2325-2337.

Gross CG, Rocha-Miranda CE, Bender DB (1972) Visual properties of neurons in inferotemporal cortex of the Macaque. Journal of neurophysiology 35:96-111.

Hanke M, Halchenko YO, Sederberg PB, Hanson SJ, Haxby JV, Pollmann S (2009) PyMVPA: a Python Toolbox for Multivariate Pattern Analysis of fMRI Data. Neuroinformatics 7:37-53.

Haxby JV, Hoffman EA, Gobbini MI (2000) The distributed human neural system for face perception. Trends in cognitive sciences 4:223-233.

Haxby JV, Gobbini MI, Furey ML, Ishai A, Schouten JL, Pietrini P (2001) Distributed and overlapping representations of faces and objects in ventral temporal cortex. Science 293:2425-2430.

Haynes JD, Rees G (2006) Decoding mental states from brain activity in humans. Nat Rev Neurosci 7:523-534 
Heeger DJ, Huk AC, Geisler WS, Albrecht DG (2000) Spikes versus BOLD: what does neuroimaging tell us about neuronal activity? Nat Neurosci 3:631-633.

Heeger DJ, Ress D (2002) What does fMRI tell us about neuronal activity? Nat Rev Neurosci $3(2): 142-151$

Hubel DH, Wiesel TN (1962) Receptive fields, binocular interaction and functional architecture in the cat's visual cortex. The Journal of physiology 160:106-154.

Hubel DH, Wiesel TN (1965) Receptive Fields and Functional Architecture in Two Nonstriate Visual Areas (18 and 19) of the Cat. Journal of neurophysiology 28:229-289.

Hung CP, Kreiman G, Poggio T, DiCarlo JJ (2005) Fast readout of object identity from macaque inferior temporal cortex. Science 310:863-866.

James W (1890) The principles of psychology. New York: H. Holt and company.

Kamitani Y, Tong F (2006) Decoding seen and attended motion directions from activity in the human visual cortex. Curr Biol 16:1096-1102.

Kanwisher N (2010) Functional specificity in the human brain: A window into the functional architecture of the mind. Proceedings of the National Academy of Sciences of the United States of America 107:11163-11170.

Kanwisher N, McDermott J, Chun MM (1997) The fusiform face area: A module in human extrastriate cortex specialized for face perception. Journal of Neuroscience 17:43024311.

Lades M, Vorbruggen JC, Buhmann J, Lange J, Vandermalsburg C, Wurtz RP, Konen W (1993) Distortion Invariant Object Recognition in the Dynamic Link Architecture. leee T Comput 42:300-311.

Logothetis NK, Pauls J, Augath M, Trinath T, Oeltermann A (2001) Neurophysiological investigation of the basis of the fMRI signal. Nature 412:150-157.

Liu T, Pestilli F, Carrasco M (2005) Transient attention enhances perceptual performance and FMRI response in human visual cortex. Neuron 45:469-477.

Miller EK, Li L, Desimone R (1991) A neural mechanism for working and recognition memory in inferior temporal cortex. Science 254:1377-1379.

Mur M, Bandettini PA, Kriegeskorte N (2009) Revealing representational content with patterninformation fMRI--an introductory guide. Social cognitive and affective neuroscience 4:101-109.

Naselaris T, Kay KN, Nishimoto S, Gallant JL (2011) Encoding and decoding in fMRI. Neuroimage 56:400-410.

Nestor A, Plaut DC, Behrmann M (2011) Unraveling the distributed neural code of facial identity through spatiotemporal pattern analysis. Proceedings of the National Academy of Sciences of the United States of America 108:9998-10003.

Olman CA, Ugurbil K, Schrater P, Kersten D (2004) BOLD fMRI and psychophysical measurements of contrast response to broadband images. Vision Res 44:669-683.

Pasupathy A, Connor CE (2002) Population coding of shape in area V4. Nat Neurosci 5:13321338.

Pelli, D. G. (1997). The VideoToolbox software for visual psychophysics: Transforming numbers into movies. Spatial vision 10:437-442.

Pinsk MA, DeSimone K, Moore T, Gross CG, Kastner S (2005) Representations of faces and body parts in macaque temporal cortex: A functional MRI study. Proceedings of the National Academy of Sciences of the United States of America 102:6996-7001.

Sclar G, Maunsell JHR, Lennie P (1990) Coding of Image-Contrast in Central Visual Pathways of the Macaque Monkey. Vision Res 30:1-10.

Tootell RBH, Reppas JB, Kwong KK, Malach R, Born RT, Brady TJ, Rosen BR, Belliveau JW (1995) Functional-Analysis of Human Mt and Related Visual Cortical Areas Using Magnetic-Resonance-Imaging. Journal of Neuroscience 15:3215-3230. 
Tong F, Pratte MS (2012) Decoding patterns of human brain activity. Annual review of psychology 63:483-509.

Tong F, Harrison S, Dewey J, Kamitani Y (2012) Relationship between BOLD amplitude and pattern classification of orientation-selective activity in the human visual cortex. Neuroimage, 63: 1212-1222.

Treisman AM, Gelade G (1980) A feature-integration theory of attention. Cognitive psychology 12:97-136.

Tsao DY, Freiwald WA, Knutsen TA, Mandeville JB, Tootell RBH (2003) Faces and objects in macaque cerebral cortex. Nat Neurosci 6:989-995.

Tsao DY, Freiwald WA, Tootell RBH, Livingstone MS (2006) A cortical region consisting entirely of face-selective cells. Science 311:670-674.

Willenbockel V, Sadr J, Fiset D, Horne GO, Gosselin F, Tanaka JW (2010) Controlling low-level image properties: the SHINE toolbox. Behavior research methods 42: 671-684.

Yamins DL, Hong H, Cadieu CF, Solomon EA, Seibert D, DiCarlo JJ (2014) Performanceoptimized hierarchical models predict neural responses in higher visual cortex. Proceedings of the National Academy of Sciences of the United States of America 111:8619-8624.

Yue X, Cassidy BS, Devaney KJ, Holt DJ, Tootell RB (2011) Lower-level stimulus features strongly influence responses in the fusiform face area. Cerebral cortex 21:35-47. 
A

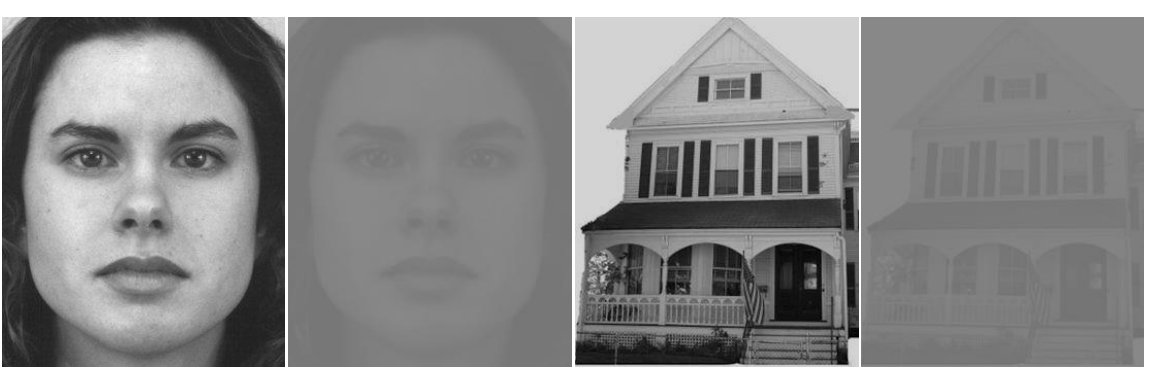

B
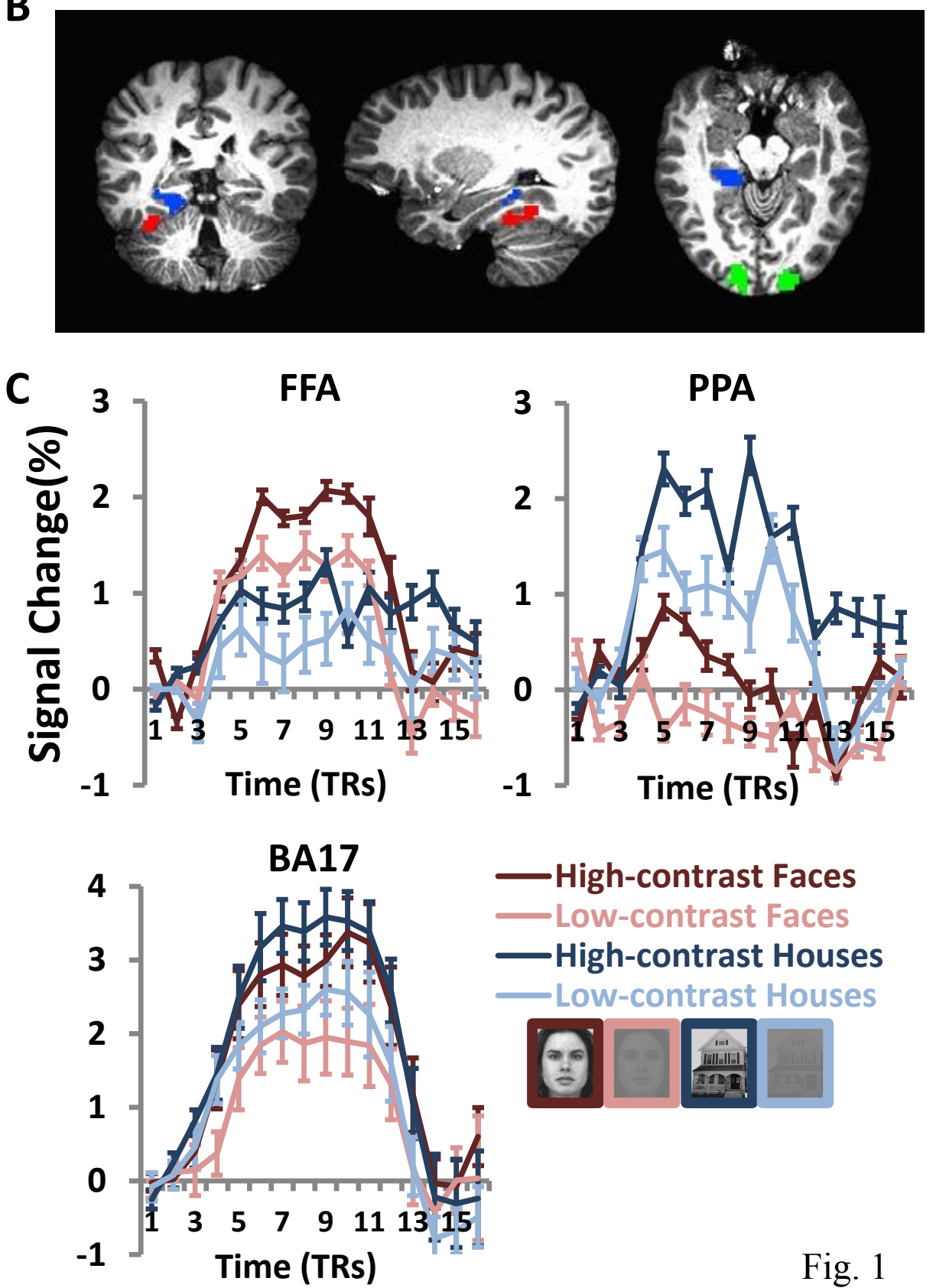

—High-contrast Faces Low-contrast Faces High-contrast Houses Low-contrast Houses

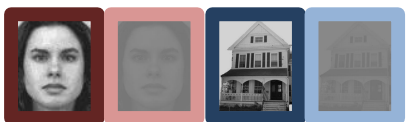

Fig. 1 
FFA

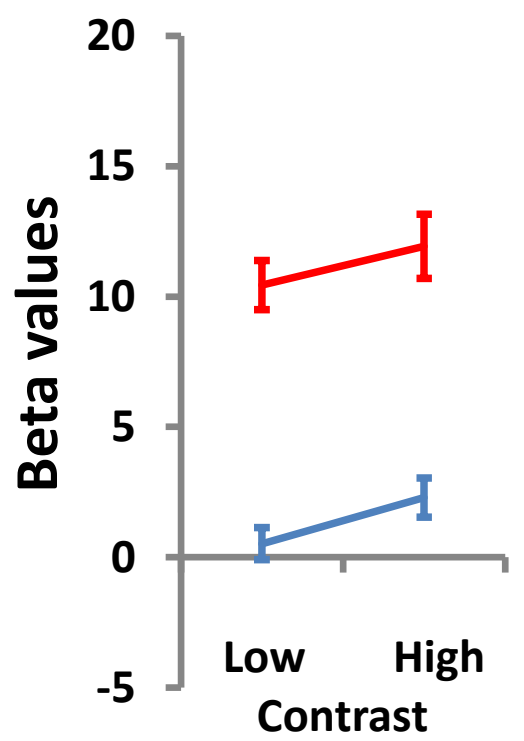

PPA

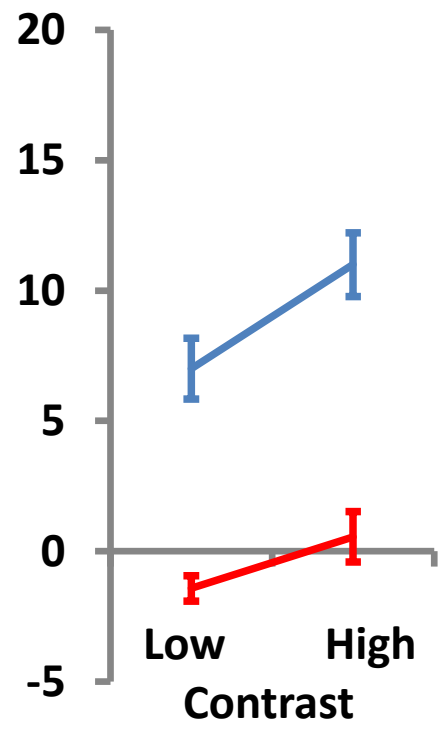

BA17

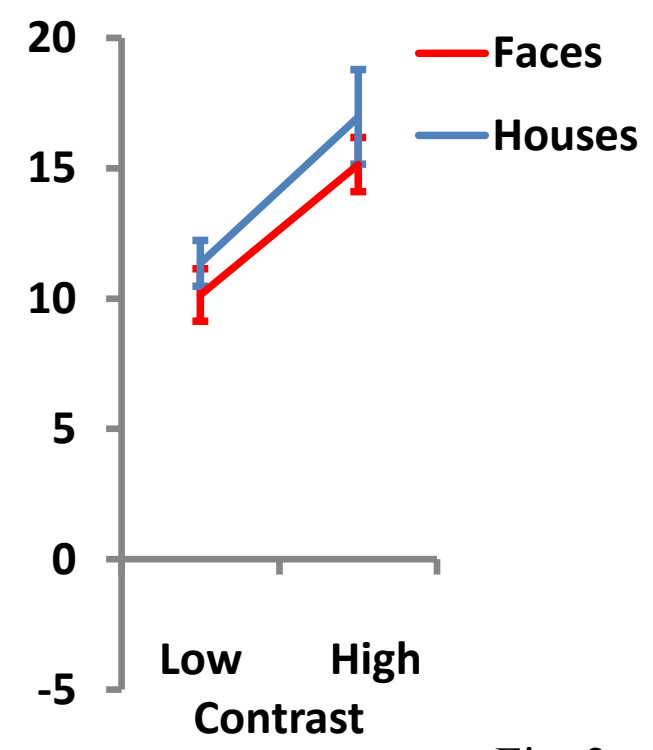

Fig. 2 
A

\section{Targets}

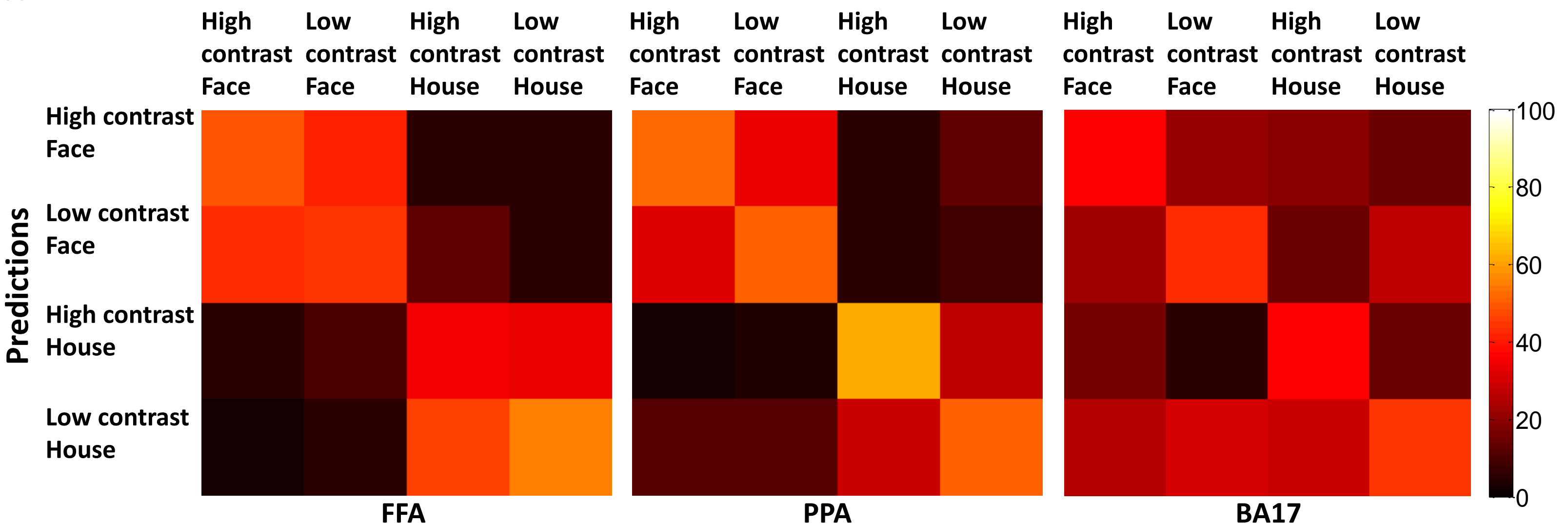

B

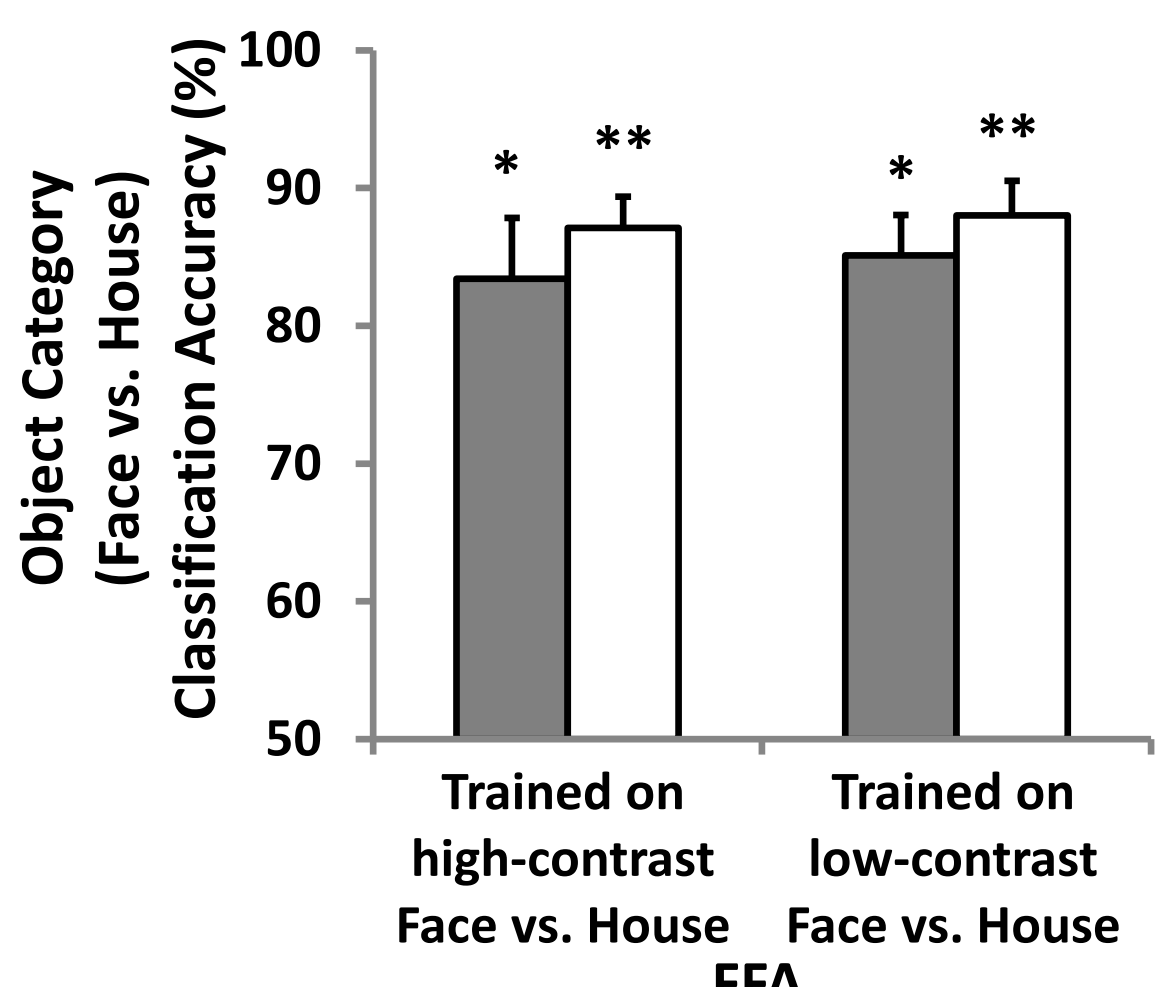

C

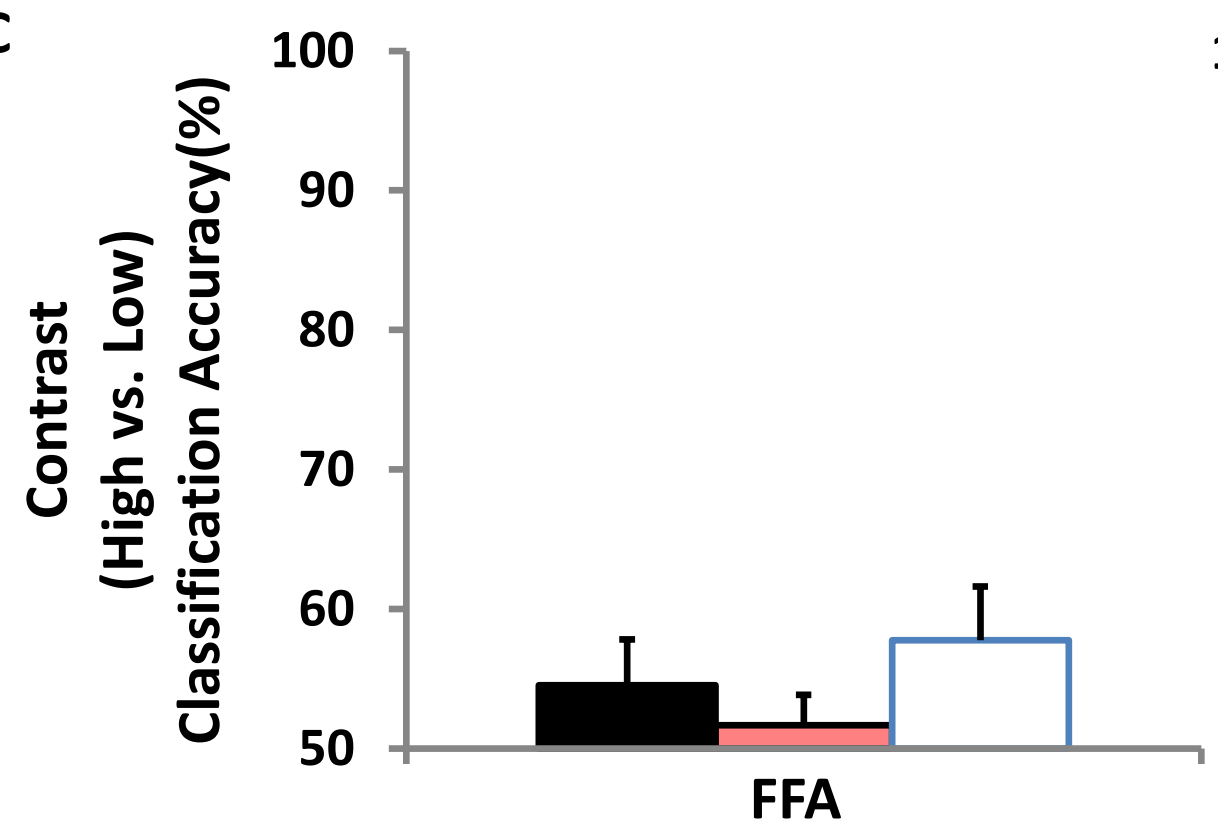

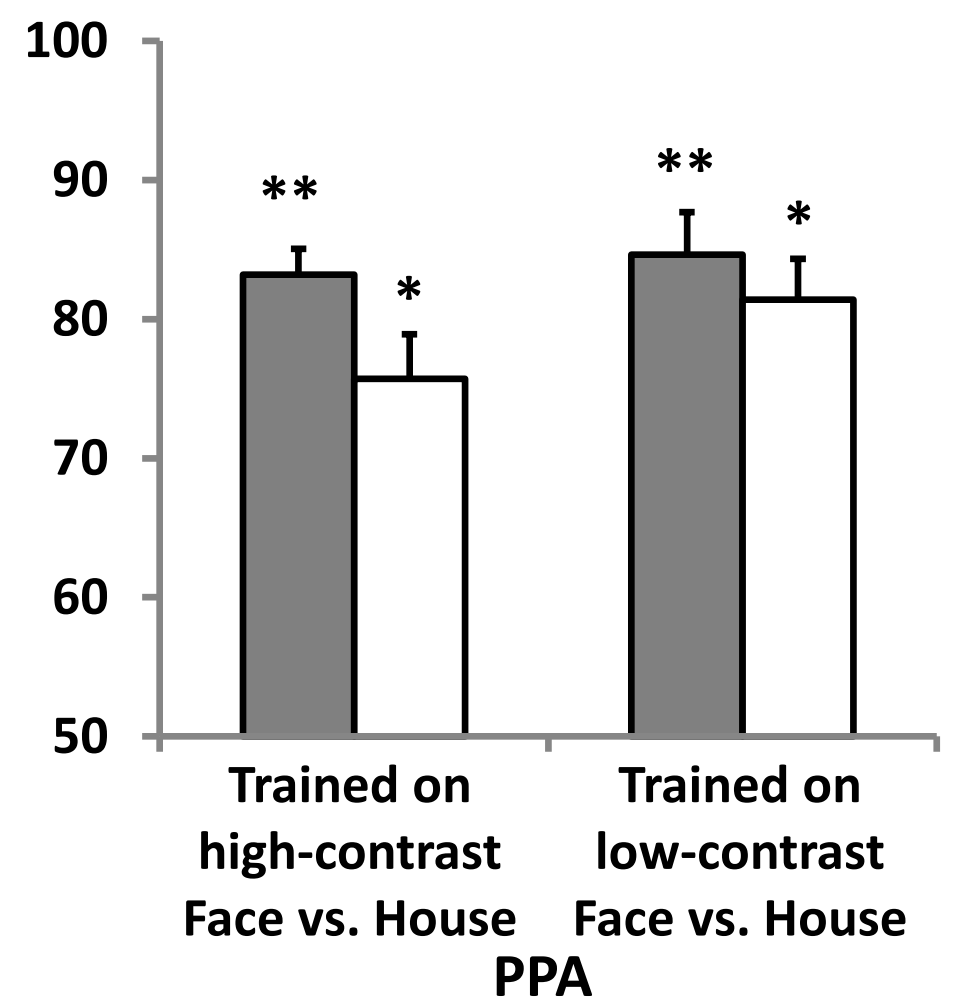
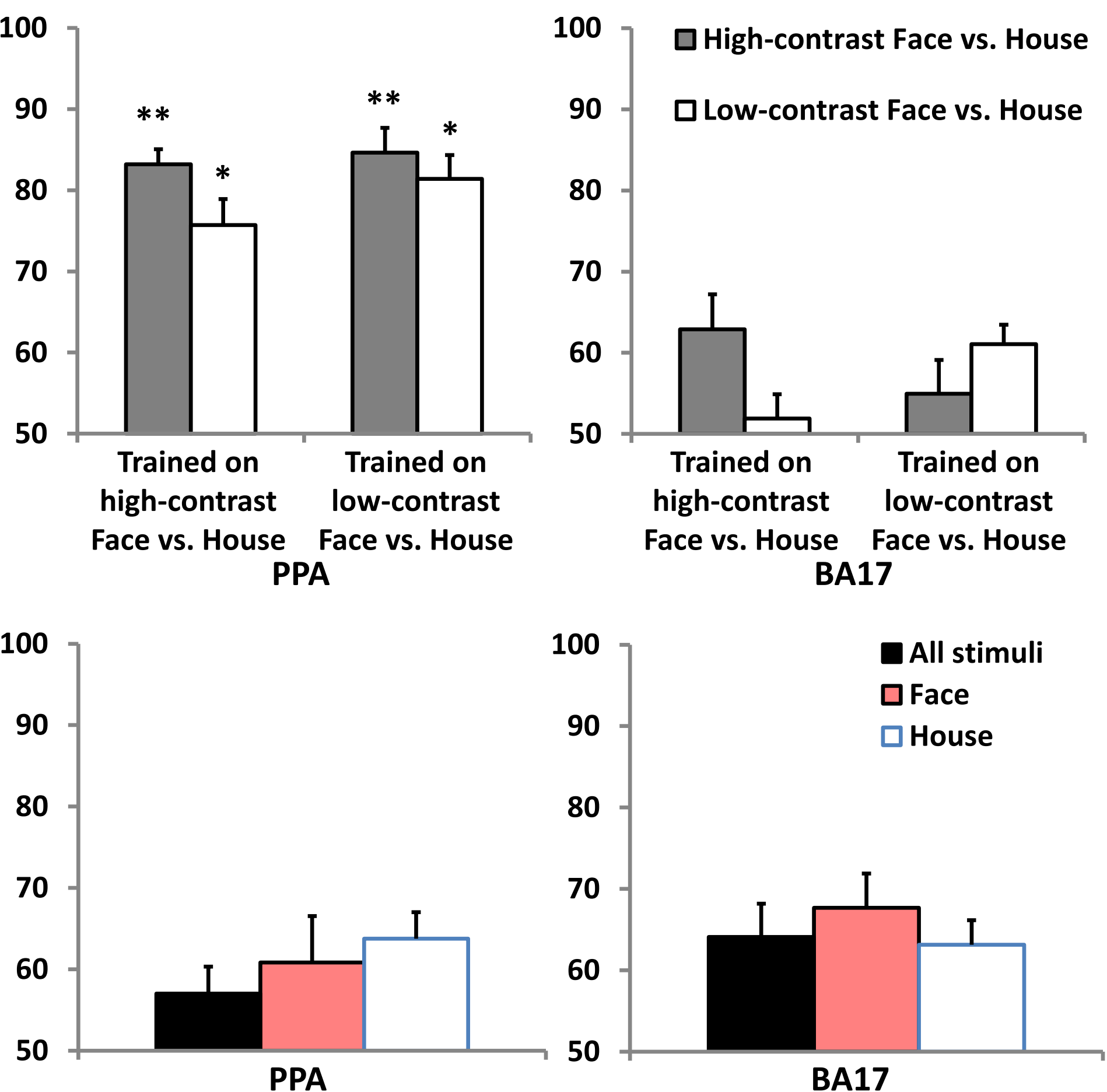

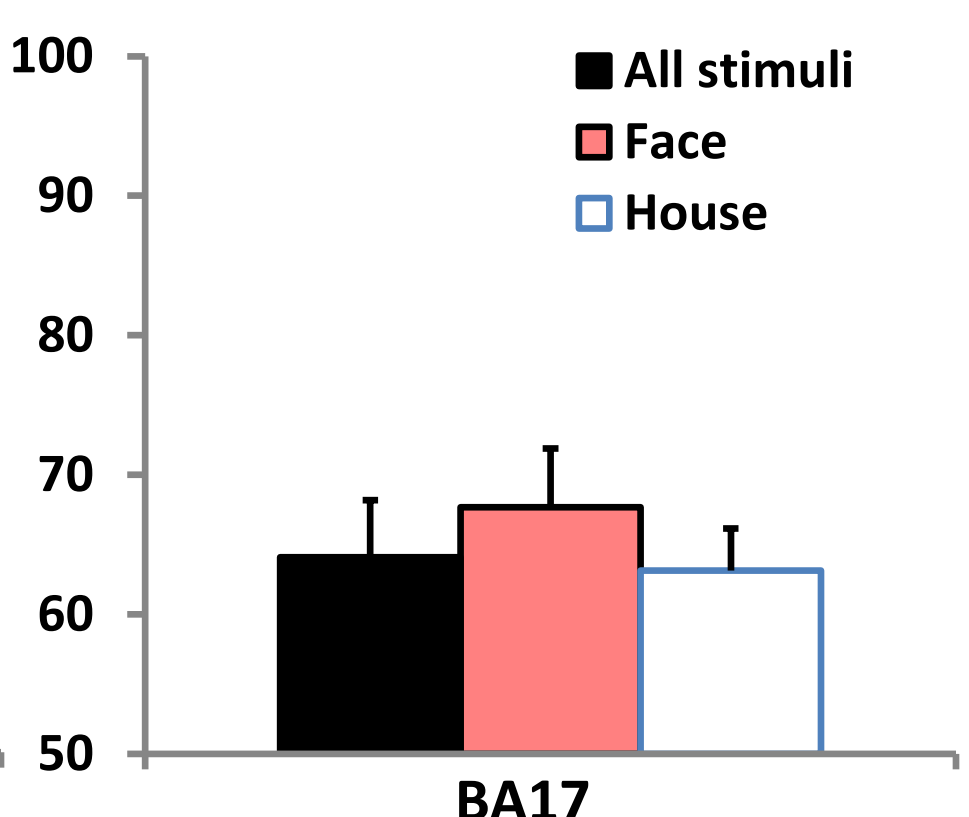


The effect of contrast

A

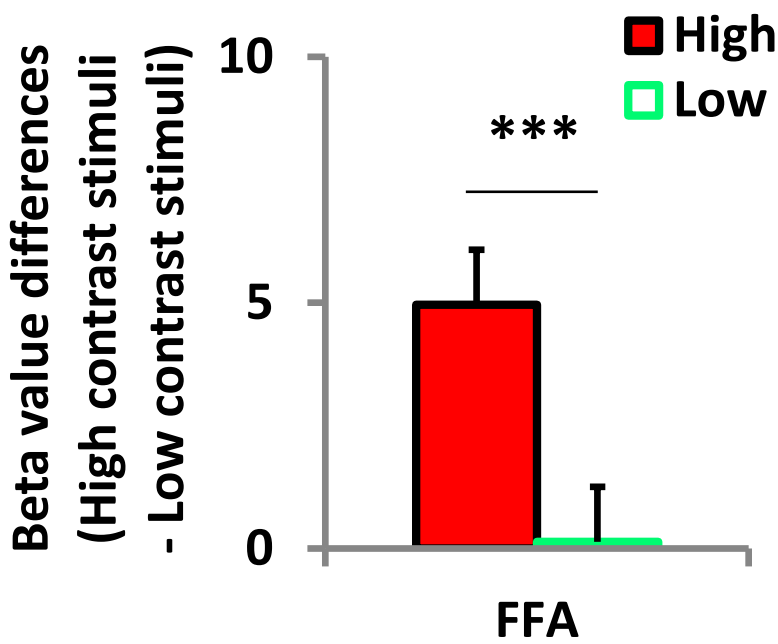

B

\section{Targets}

High Low High Low High Low High Low

contrast contrast contrast contrast contrast contrast contrast contrast

Face Face House House Face Face House House
High contrast

Face

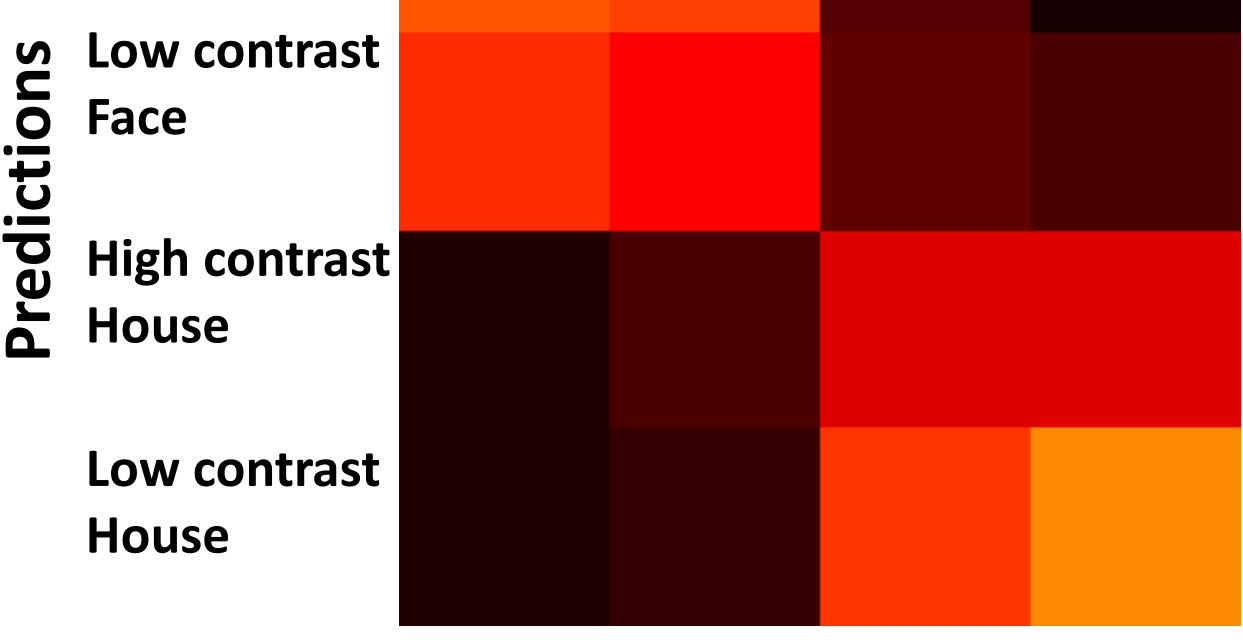

HCFFA
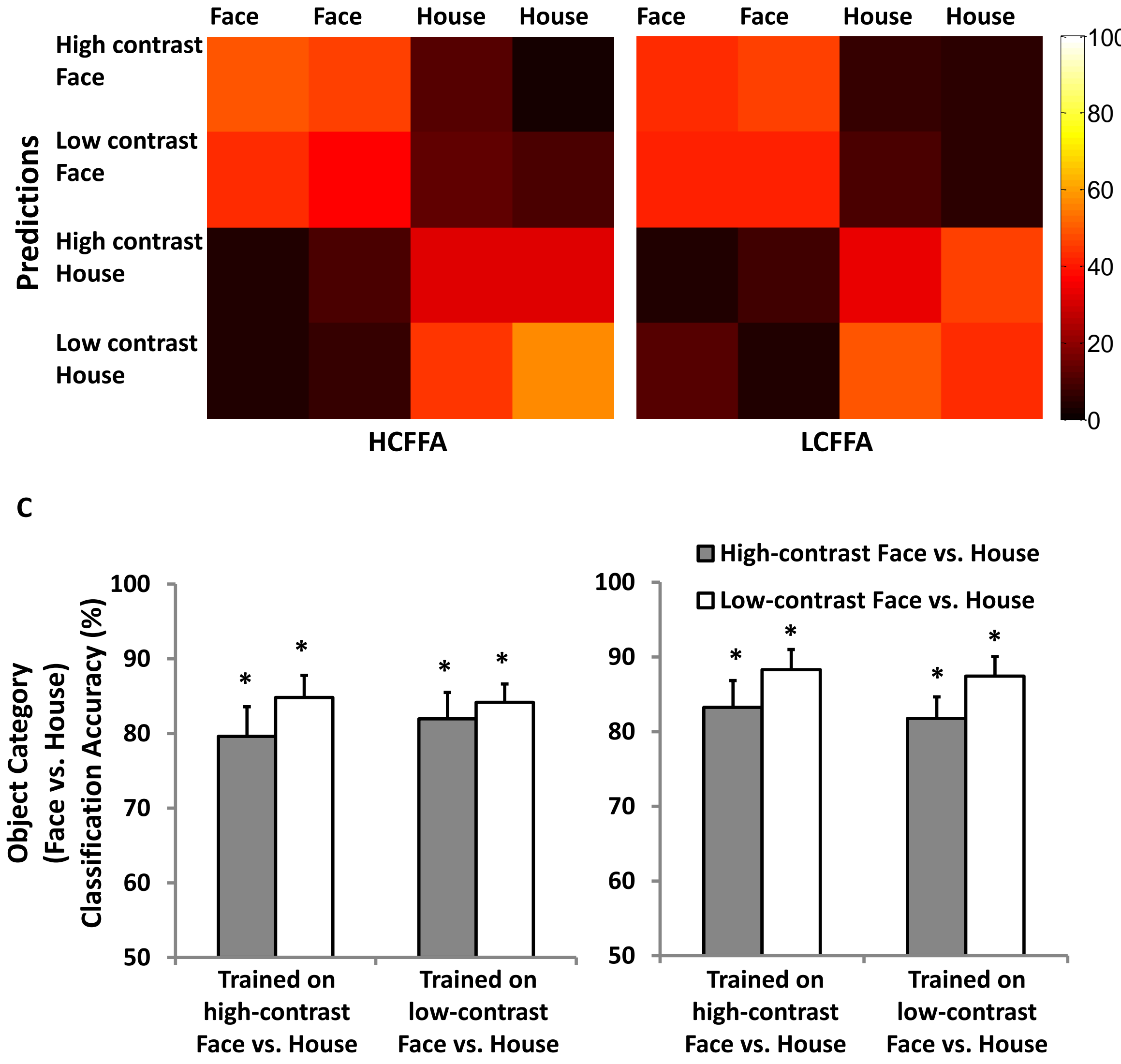

LCFFA
The effect of contrast
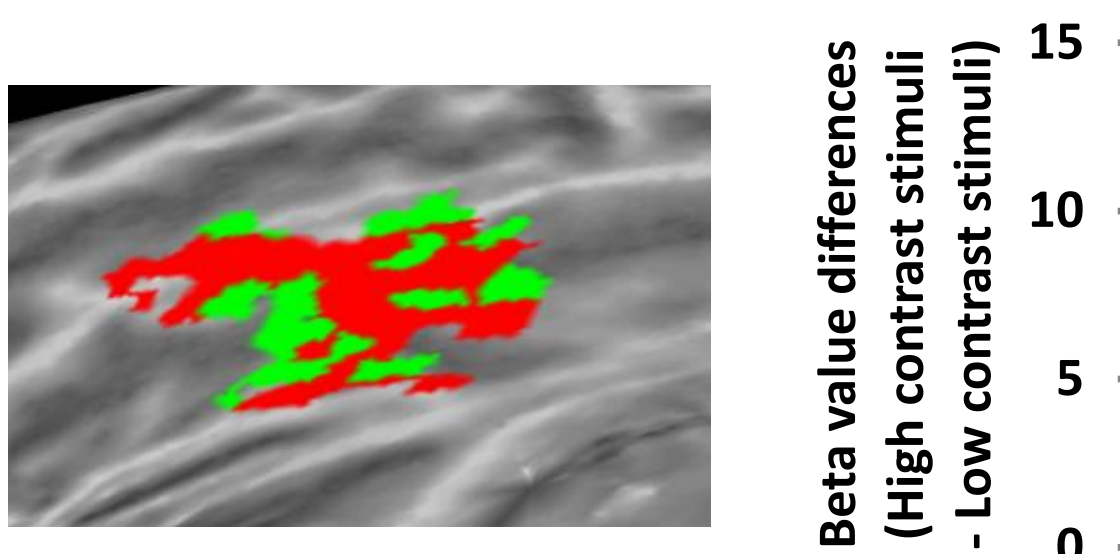

High sensitive 50 voxels *** $\square$ Low sensitive 50 voxels

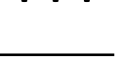

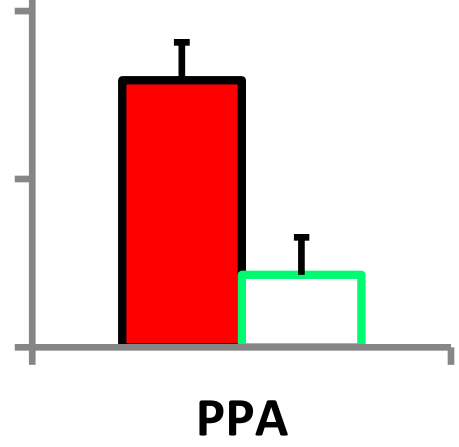

$\mathbf{E}$

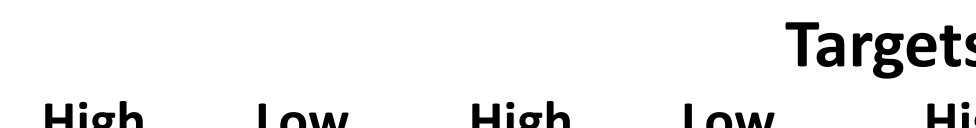

High Low High Low High Low High Low contrast contrast contrast contrast contrast contrast contrast contrast Face Face House House Face Face House House

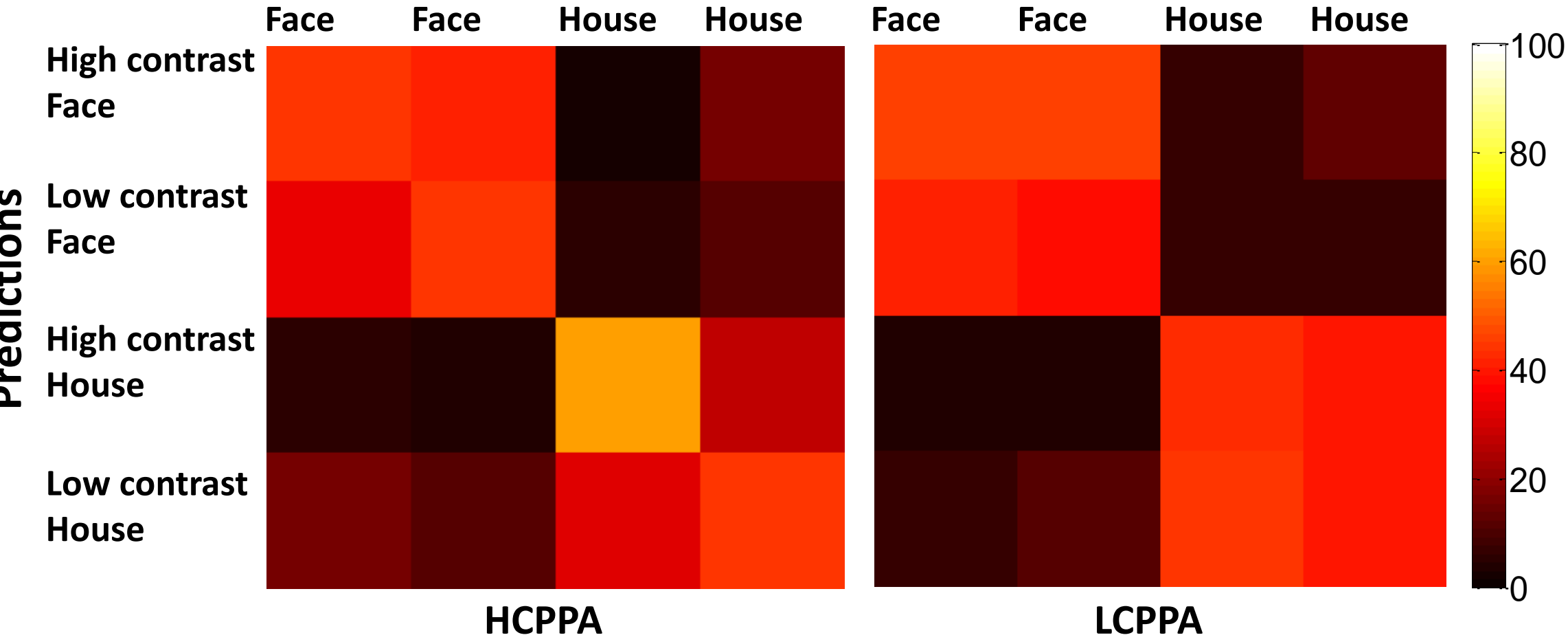

$\mathbf{F}$
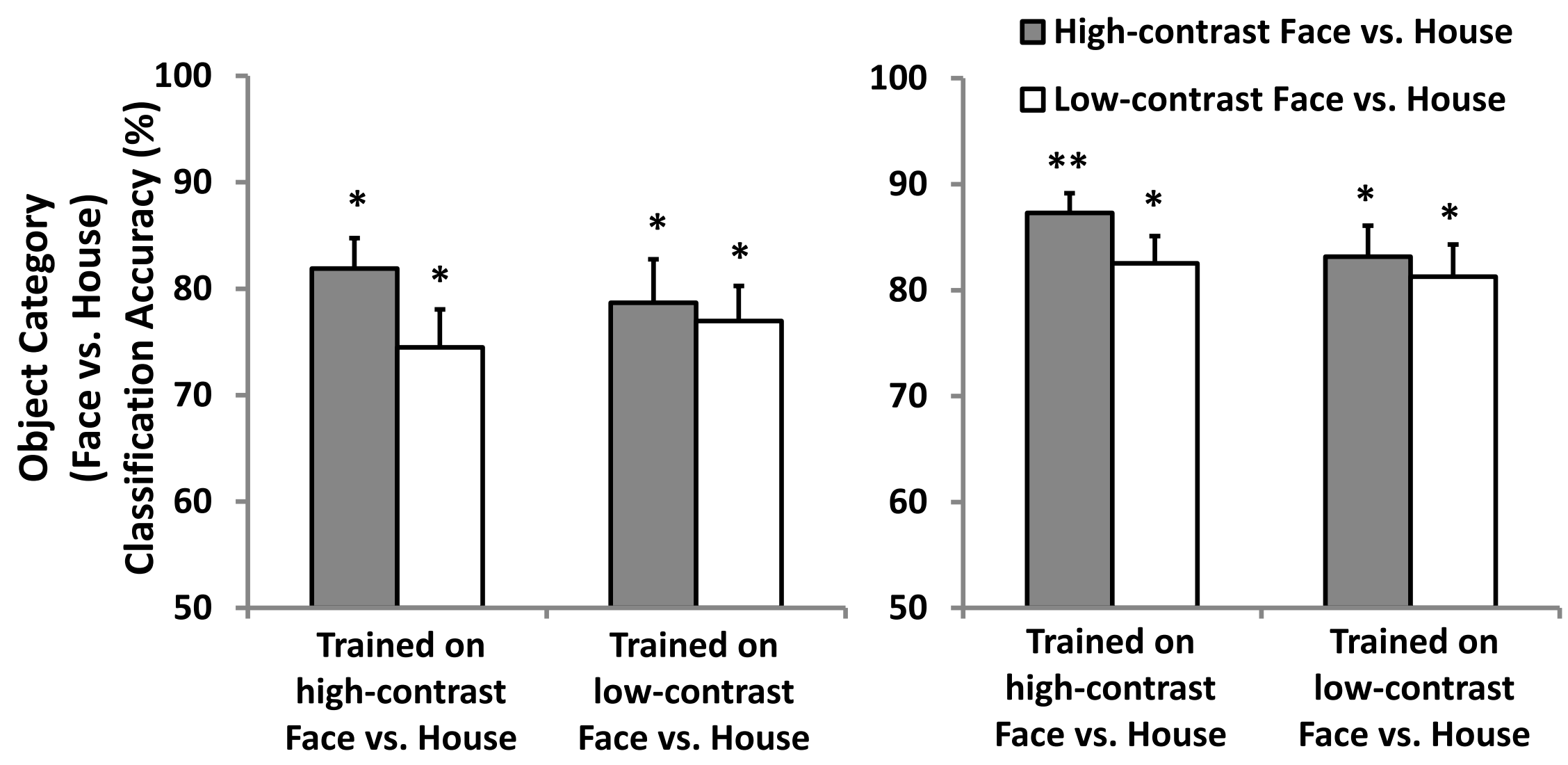

HCPPA 


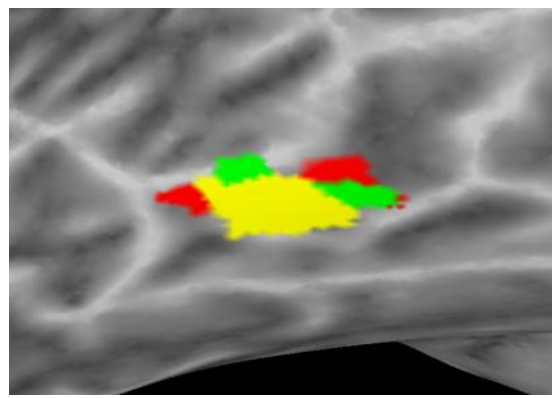

FFA

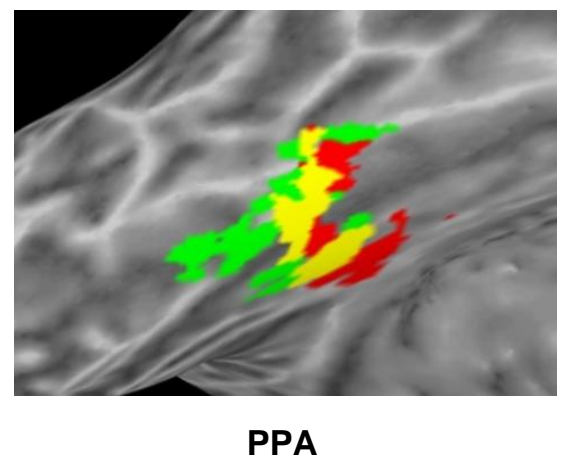

Fig. 5 